\title{
Three in One: Case Report Supporting Different Origins of Essential and Parkinsonian Tremors
}

\author{
G. Linazasoro $^{a}$ N. Van Blercom ${ }^{a}$ C. Magariños ${ }^{b}$

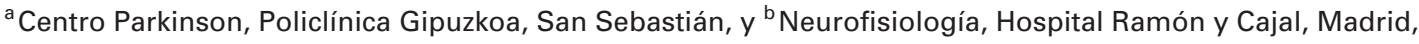 \\ España
}

Dear Sir,

There are various 'oscillation generators' in the central nervous system whose activity could provoke tremors [1]. Among them, the ventralis intermedius (VIM) nucleus of the thalamus, the cerebellar dentate and red nuclei, and the inferior olive are the most relevant, constituting the olivo-cerebello-thalamic circuits [1]. Neuronal oscillatory activity may also be generated in the basal ganglia, as has been demonstrated in Parkinson's disease (PD) [1]. All these nuclei are interconnected. Thus their oscillatory activity could converge at the VIM level, which explains the remarkable efficacy of thalamic surgery against all types of tremor [2]. However, there could be independent oscillators according to the phenomenology and somatotopy of the tremor [1]. We report on the case of a patient who presented tremors of diverse etiology, showing different responses to different therapeutic approaches and thus supporting this last possibility.

\section{Case Report}

A 66-year-old woman was sent to our hospital to evaluate stereotactic surgery because of a 20-year history of severe tremor refractory to medical treatments. She had a family history of tremor. The tremor began affecting both upper extremities when maintaining a posture, and its severity was gradually increasing. The first clinical examination at our center revealed rest and postural tremors in both upper and lower extremities [3-4/4 on the motor subscale of the Unified PD Rating Scale (UPDRS) [3]], a severe truncal tremor when standing (phenomenologically similar to symptomatic orthostatic tremor) and very mild bradykinesia in the left hemibody. Propranolol (up to $120 \mathrm{mg} /$ day), nadolol (up to 80 ), primidone (up to 750), gabapentin (up to 2,400 ) and clonazepam (up to 6) were tried, alone and in different combinations, without success. Since we also found a very mild parkinsonism, we performed a levodopa test with $300 \mathrm{mg}$, which failed to produce any improvement. Other causes of tremor (including hyperthyroidism) were reasonably ruled out, and the cerebral MRI was normal. A single-proton emission computed tomography scan to study the functional situation of the presynaptic dopaminergic nigrostriatal pathway was not performed.

Based on the clinical evolution and the lack of response to a high dose of levodopa, a diagnosis of severe ET (essential tremor) was established, and a bilateral deep brain stimulation (DBS) of the VIM thalamic nucleus rather than a DBS of the subthalamic nucleus (STN) was indicated. The procedure was performed with microelectrodes, and numerous tremoric neurons were recorded, most of them related to the arms. The upper tremor disappeared, and the lower one was partially improved when the corresponding areas were stimulated with the recording microelectrode. The truncal tremor could not be evaluated in the operat- ing room. While the implantation of the definitive electrodes yielded a perfect control of the tremor of the arms, it only led to a mild improvement in the ones of the legs and the trunk, despite the use of different combinations of contact electrodes (from mono- to bi- and tripolar) and high voltages (up to $5 \mathrm{~V}$ ), which induced dysarthria. The stimulation parameters required for controlling the postural tremor of the arms were amplitude $(3.5 \mathrm{~V})$, pulse width $(90 \mu \mathrm{s})$ and rate $(185 \mathrm{~Hz})$ using monopolar stimulation with contact 2 cathodal and the case anodal.

In the following 2 months a clear-cut parkinsonism was evident, scoring 23 on the motor subscale of the UPDRS. Indeed, the tremors in the legs and trunk were totally abolished for $>2 \mathrm{~h}$ with the intake of $500 \mathrm{mg}$ of levodopa. The treatment with levodopa (500 mg t.i.d.) and pramipexole (1.5 mg t.i.d.) was effective. Switching off the stimulators during the peak effect of levodopa led to a reappearance of postural tremor in the arms to the same extent as seen preoperatively. It can thus be considered that the arm tremor was levodopa unresponsive and likely related to severe ET. A few weeks later the patient began complaining of loss of appetite, nausea, loss of weight, nervousness and profuse sweating, which was attributed to the intolerance of the antiparkinsonian medication. However, a blood analysis revealed a severe hyperthyroidism related to a hyperfunctioning

\section{KARGER}

Fax +4161306 1234 E-Mail karger@karger.ch www.karger.com
(C) 2006 S. Karger AG, Basel 0014-3022/06/0552-0108\$23.50/0

Accessible online at: www.karger.com/ene
G. Linazasoro

Centro de Investigación Parkinson, Policlínica Gipuzkoa

Parque Tecnológico de Miramón

ES-20009 San Sebastián, Gipuzkoa (Spain)

Tel. +34 610406 449, Fax +34 943002 859, E-Mail glinazasoro@terra.es 
goiter. The antithyroid therapy produced a substantial improvement of most of these new symptoms. The postural tremor of the arms was not modified. The trunk and lower extremitiy tremors were improved, allowing a reduction of the dosage of levodopa to $312.5 \mathrm{mg}$ t.i.d. (11/4 tablets of Sinemet t.i.d.) and a withdrawal of pramipexole. In spite of the improvement observed after the correction of the hyperthyroidism, the patient still needed levodopa to completely abolish the tremor, as well as rigidity and bradykinesia. A wearing-off effect was evident 3 months later. It was characterized by a reemergence of severe leg and truncal tremors when standing, accompanied by autonomic (e.g. profuse sweating or facial rubor) and emotional (anxiety and depressive mood) symptoms when the effects of a dosage of levodopa vanished. Further increases in the dosage of levodopa and the reintroduction of pramipexole were not tolerated well. Therefore a bilateral STN stimulation was planned. However, it could not be performed eventually, since the patient's cognitive state had been deteriorating. At present, she is in a similar clinical situation, with a well-controlled postural tremor of the arms, parkinsonism with motor and nonmotor wearing-off, and a moderate cognitive impairment.

\section{Discussion}

Our patient suffered from 3 possible causes of tremor (ET, PD and hyperthyroidism). It seems clear that at least 2 independent oscillators were responsible for the postural tremor in the upper extremities, and for the truncal and leg tremors, respectively. The former was improved by thalamic surgery, thus suggesting the participation of the VIM thalamic nucleus in its origin. Levodopa similarly improved the latter by a mechanism involving the STN: levodopa, apomorphine and STN stimulation improve parkinsonian motor signs by reducing the firing rate of STN neurons in animal models of PD as well as in patients [4-6; pers., unpubl. data].

This observation contradicts the findings reported by Murata et al. [7] and more recently by Stover et al. [8]. However, as STN surgery was eventually not performed in our patient, we cannot exclude the existence of further effects of STN DBS. In contrast to the present observation, numerous studies have shown that VIM stimulation is equally effective in parkinsonian (arm and leg) and essential tremors. It could thus be considered that the thalamic electrodes were not implanted in an ideal area of the nucleus. The VIM nucleus is organized in a highly somatotopic way [9], which is why the electric current resulting from the DBS electrode did probably not inhibit the oscillatory activity accounting for the tremor affecting the trunk and lower extremities. This possibility seems quite remote, since numerous tremor-synchronous activities were found during the procedure, which included an exhaustive mapping of the nucleus. Additionally, microstimulation produced a partial improvement of the leg tremor. Furthermore, the time course of parkinsonism during the next months argues against this possibility.

Our patient developed a quite severe parkinsonian syndrome 2 months after the thalamic surgery, complicated by a wearing-off phenomenon 4 months after the introduction of levodopa. The emergence of parkinsonism might have been caused by the surgical procedure itself or by the severe hyperthyroidism, which could aggravate a preexisting mild $\mathrm{PD}$ as has been previously reported $[10,11]$.

\section{References}

1 Elble RJ: Origins of tremor. Lancet 2000;355: 1113-1114.

2 Pahwa R, Lyons K, Koller WC: Surgical treatment of essential tremor. Neurology 2000; 54(suppl 4):S39-S44.

3 Martínez Martín P: Rating scales in Parkinson's disease; in Tolosa E, Jankovic J (eds): Parkinson's Disease and Movement Disorders. Baltimore, Williams \& Wilkins, 1993, pp 281-292.

4 Kreiss DS, Mastropietro CW, Rawji SS, Walters JR: The response of subthalamic nucleus neurons to dopamine receptor stimulation in a rodent model of Parkinson's disease. J Neurosci 1997; 17:6807-6819.

5 Rodríguez MC, Gorospe A, Linazasoro G, et al: Bilateral deep brain stimulation of the subthalamic nucleus in Parkinson's disease. Neurology 2000;55(suppl 6):45-51.

6 Dostrovsky JO, Lozano AM: Mechanisms of deep brain stimulation. Mov Disord 2002; 17(suppl 3):S63-S68.

7 Murata J, Kitagawa M, Uesugi H, et al: Electric stimulation of the posterior subthalamic area for the treatment of intractable proximal tremor. J Neurosurg 2003;99:708-715.

8 Stover NP, Okun S, Evatt ML, Raju DV, Bakay RAE, Vitek JL: Stimulation of the subthalamic nucleus in a patient with Parkinson disease and essential tremor. Arch Neurol 2005; 62:141-143

9 Vitek JL, Wichmann T, DeLong MR: Current concepts of basal ganglia pathophysiology relative to tremor genesis; in Findley LJ, Koller WC (eds): Handbook of Tremor Disorders. New York, Dekker, 1995, pp 37-50.

10 Caradoc-Davies TH: Resolution of dyskinesia and the 'on-off' phenomenon in thyrotoxic patients with Parkinson's disease after antithyroid treatment. Br Med J 1986;293:38-39.

11 Linazasoro G, Martí Massó JF: Different influences of hyperthyroidism on Parkinson's disease. Mov Disord 1992;7(suppl 1):37. 